\title{
Subchronic Effects of Ectoine on Survival, Growth and Physiological Parameters of Daphnia Magna
}

\section{Bownik Adam ${ }^{1 *}$ and Stępniewska Zofia²}

${ }^{1}$ Department of Physiology and Ecotoxicology, Faculty of Biotechnology and Environmental Sciences, The John Paul II Catholic University of Lublin, Poland ${ }^{2}$ Department of Biochemistry and Environmental Chemistry, Faculty of Biotechnology and Environmental Sciences, The John Paul II Catholic University of Lublin, Poland

\begin{abstract}
Ectoine (ECT) is an osmoprotectant produced and accumulated by halophilic bateria under hyperosmotic stress. There is a lack of knowledge on the effect induced by this amino acid during subchronic animal exposure to ECT in vivo. Therefore, the aim the present study was to determine influence of ECT on survival, motility, physiological activity (heart rate, thoracic limb activity, and mandible movement rate), growth rate and moulting of unfed and fed with normal diet Daphnia magna exposed for 10 days to $2.5,4,20$ and $25 \mathrm{mg} / \mathrm{L}$ ECT. The results showed that survival of unfed animals exposed to $25 \mathrm{mgL}$ ECT was decreased; however it was increased at lower concentrations of the amino acid when compared to the control. 20 and $25 \mathrm{mg} / \mathrm{L}$ of ECT significantly decreased swimming velocity, heart rate and thoracic limb activity and mandible movement rate. On the other hand, the osmoprotectant did'nt cause mortality of fed daphnids at any concentration but stimulated swimming velocity and the physiological parameters, however no differences were found in mandible movement rate. We found that ECT increased growth rate both in starving and fed daphnids, however the stimulatory effect was not concentation-dependent. The most significant increase of growth rate both in unfed and fed dapnids was found at $4 \mathrm{mg} / \mathrm{L} \mathrm{ECT}$. Moulting frequency was increases only in unfed daphnids exposed to the highest concentrations of ECT. Fed daphnids showed slighlty elevated moulting frequency only at $25 \mathrm{mg} / \mathrm{L}$ ECT. Our results indicate ECT is a stimulator of growth rate and physiological indices in fed Daphnia magna, however the elucidation of its mechanisms requires further studies.
\end{abstract}

Keywords: Ectoine; Daphnia magna; Growth; Swimming velocity; Heart rate; Thoracic limb movement

\section{Introduction}

Some amino acids like proline, glutamate, glutamine, alanine, ectoine, hydroxyectoine) and their derivatives belong to a group of compounds known as "compatible solutes". These low molecular weight substances are produced and accumulated in bacteria, algae for protection against stressful factors [1]. Compatible solutes are known to protect phospholipids and proteins against denaturation and dehydration without interference with cellular processes [2-4].

Ectoine (ECT) (1,4,5,6-tetrahydro-2-methyl-4-pyrimidine carboxylic acid) is a compatible solute produced by aerobic, chemoheterotrophic, and halophilic bacteria to survive under extreme conditions [5]. Microorganisms such as Marinococcus may synthesize and accumulate intracellular ECT in response to osmotic changes in the environment [6]. Production of ECT was also reported in Vibrio cholerae which may temporarily occur in some aquatic environments adapting to changes in osmolarity [7], Bacillus sp. and gastroenteritic Vibrio parahaemolyticus under hyperosmotic conditions [8,9]. ECT was demonstrated to enhance stability of different thermolabile molecules, such as phytase, lactic dehydrogenase (LDH) and phosphofructokinase (PFK), [10-13]. This amino acid has also been proposed as an alternative to dimethyl sulfoxide for cryopreservation of various cells $[14,15]$. It is speculated that since ectoine is a water-binding amino acid, it may increase the level hydration of various molecules and thereby improve their stabilization during osmotic stress. Higher level of fluidity may be advantageous to cell membranes to cope with extreme conditions like high or low temperatures, changing osmotic pressure and may accelerate repair mechanisms in some cells [16].

Protective effects of ECT were found in animals. Our previous study showed protective effects of ECT in Daphnia magna subjected to heat stress [17]. We also showed low toxicity of ECT to these cladocerans during short-term exposure [18] however, no data are available on subchronic effects of this osmoprotectant in animals subjected to its lower concentrations. Some amino acids classified as compatible solutes such as taurine turned out to stimulate growth of some aquatic animal species $[19,20]$ therefore we hypothesized that ECT may also induce similar effects in cladocerans. We selected Daphnia magna as an animal model since it is commonly used in toxicological studies and has valuable features, such as high sensitivity, transparency of the carapace and a short time of reproduction. The aim of our study was to determine survival, swimming behaviour, heart rate, thoracic limb activity, mandible movement rate, growth and moulting of Daphnia magna during subchronic exposure to ECT.

\section{Material and Methods}

\section{Culture method}

Daphnia magna were cultured in a continuous reproduction for a few months in several $6 \mathrm{~L}$ tanks with $5 \mathrm{~L}$ of aerated culture medium on the window ledge in a laboratory under light: dark period of $16 \mathrm{~h}: 8 \mathrm{~h}$. Daphnia culture medium was prepared following the ASTM standards (American Society of Testing and Materials, 1986). The medium was synthetic freshwater $\left(48 \mathrm{mg}\right.$ of $\mathrm{NaHCO}_{3}, 30 \mathrm{mg}$ of $\mathrm{CaSO}_{4} \cdot 2 \mathrm{H}_{2} \mathrm{O}, 30$ $\mathrm{mg}$ of $\mathrm{MgSO}_{4}$ and $2 \mathrm{mg}$ of $\mathrm{KCl}$ per liter of deionized water adjusted to a $\mathrm{pH}$ of 7.4), with a temperature of $23 \pm 2^{\circ} \mathrm{C}$. The number of cultured

*Corresponding author: Bownik Adam, Department of Physiology and Ecotoxicology, Faculty of Biotechnology and Environmental Sciences, The John Paul II Catholic University of Lublin, Poland; E-mail: adambownik@wp.pl

Received March 23, 2015; Accepted May 13, 2015; Published June 30, 2015

Citation: Adam B, Zofia S (2015) Subchronic Effects of Ectoine on Survival, Growth and Physiological Parameters of Daphnia Magna. J Aquac Res Development 6: 352. doi:10.4172/2155-9546.1000352

Copyright: (C) 2015 Adam B, et al. This is an open-access article distributed under the terms of the Creative Commons Attribution License, which permits unrestricted use, distribution, and reproduction in any medium, provided the original author and source are credited. 
daphnids was about 30 animals per liter. The animals were fed once daily with a few drops of powdered Spirulina ( $2 \mathrm{mg} / \mathrm{L}$ water) per tank and supplemented with a few drops per tank of stock suspension of baker's yeast ( $10 \mathrm{mg} / \mathrm{L}$ water). Daphnids selected randomly were not fed for 24 hours prior to their use in the starvation experiment.

\section{Ectoine concentrations and experimental setup}

Pure ectoine (ECT) standard (Sigma-Aldrich), $\geq 99 \%$ purity produced by Halomonas elongata was diltuted in Daphnia magna culture medium to reveal concentrations of $2.5,4,20$ and $25 \mathrm{ml} / \mathrm{L}$ selected on the basis of its acute toxicity [18]. Neonate $24 \mathrm{~h}$ old were treated with different concentrations of ECT and animals that were not treated with ECT were maintained in clean medium only. Twothirds of each ECT solution were replaced every $72 \mathrm{~h}$. Two groups of daphnids: unfed (for starvation experiment) and fed once daily with normal diet, $10 \mu \mathrm{l}(2 \mathrm{mg} / \mathrm{L})$ and supplemented with $10 \mu \mathrm{l}$ of baker's yeast $(10 \mathrm{mg} / \mathrm{L})$ per 10 dapnids kept in $100 \mathrm{ml}$ of medium were exposed to the appropriate concentrations of ECT. Individual feeding was performed in growth rate experiment.

\section{Determination of Daphnia magna survival}

10 daphnids (in 3 replicates) were placed in $150 \mathrm{ml}$ glass beakers containing $100 \mathrm{ml}$ of medium with appropriate concentration of ECT. Survival of both unfed and fed animals was determined every 24 hours of the exposure. The experimental animals were treated as dead when no heart beat was noted during examination under a light microscope.

\section{Swimming velocity}

Swimming velocity of Daphnia magna neonates was determined by the method described by Shimizu et al. with some modifications. 10 animals (in three replicates) were transferred at appropriate times from one the experimental group to the observation plastic dish of $55 \mathrm{~mm}$ diameter containing $6 \mathrm{ml}$ of the same concentration of ECT. Swimming daphnids in each dish were video recorded for a minimum of 1 minute (with a speed of 30 frames/second) with a digital camera Nikon D3100 mounted on a stand over the dish. Vertical movement of Daphnia was negligible because of very small depth of the solution present in the dish. All daphnids returned to the experimental beakers for further exposure after the examination. The video file with the recorded trajectories of swimming animals was analyzed by a frame-by-frame method with Tracker ${ }^{\oplus}$. By clicking with the cursor on Daphnia image in separate frames, the program plotted the whole trail left by an individual Daphnia (interpreted by the program as a mass point) measuring its maximal, minimal and mean velocity (v) expressed in milimeters per second $(\mathrm{mm} / \mathrm{s})$. Since the experimental animals moved virtually only in two dimensions swimming behaviour analysis was based on the trajectory represented by $\mathrm{x}$ and $\mathrm{y}$ coordinates. The velocities of ten daphnids calculated by software were plotted in separate graphs which were then superimposed. Swimming speed was not equal for all individuals in every experimental group and the control, therefore the mean velocity (v) of 10 daphnids from each experimental group was meaned and treated as one result.

\section{Heart and mandible movement rate and thoracic limb activity}

Heart rate and thoracic limb movement were determined by light microscopy at appropriate times of the exposure. A single daphnid was transferred in a $20 \mu \mathrm{l}$ droplet with a pipette from each experimental group to a microscope slide. Movements of the examined animals were limited by cotton wool fibers placed on the slide. The microscopic view was recorded for more than 1 minute (with a speed of 30 frames per second) with a digital camera Nikon D3100 mounted on a light microscope. The magnification $(30-100 \mathrm{X})$ and camera resolution allowed to record the activity of heart and thoracic limbs with a good quality. Video analysis was done with Tracker ${ }^{\circledR}$ software by a frameby-frame method and counting separate heart contractions during 1 minute. Thoracic limb movement and mandible movement rate were also determined by a frame-by-frame video analysis and counting the separate movements (beats) of the appendages per 1 minute.

\section{Determination of growth rate and moulting}

Two groups of 10 (in 3 replicates) randomly selected neonate daphnids were used for the experiment. The animals in the two groups were placed individually in $30 \mathrm{ml}$ transparent tubes with $20 \mathrm{ml}$ medium containing appropriate concentration of ECT. The first group of the exposed animals was subjected to starvation while the second group was simulaneously fed once daily for a period of 10 days with $1 \mu \mathrm{l}$ of powdered

Spirulina $(2 \mathrm{mg} / \mathrm{L})$ and supplemented with $1 \mu \mathrm{L}$ of baker's yeast (10 $\mathrm{mg} / \mathrm{L}$ ) per individual. Daphnids in all the experimental groups were transferred to a renewed solution of ECT after $48 \mathrm{~h}$. Video recording of growing daphnids was done every $24 \mathrm{~h}$ (Figure 1). A single daphnid was taken from the tube with appropriate concentration of ECT and transferred with a $20 \mu \mathrm{l}$ droplet to a microscope slide with cotton wool fibers placed on its surface for limitation of animal movements. The microscopic view of examined daphnid was recorded with a digital camera Nikon D3100 mounted on a light microscope. Size of daphnis was measured daily with the use of a light microscope and digital images processed by Image Tool ${ }^{\circledR}$ software. Daphnia growth was determined by measurement of distance from the most anterior part of the head to the base of tail spin and its further comparison between experimental groups. Moulting success was expressed as percentage of daphnids that shed their carapaces. Number of moults was determined by counting shed carapaces and calculation of their quantity per one individual.

\section{Statistical analysis}

All results are presented as means \pm SD. Data were analysed by one-way analysis of variance (ANOVA) followed by Duncan range test $(\alpha=0.05)$. Experimental data were processed using ANOVA followed

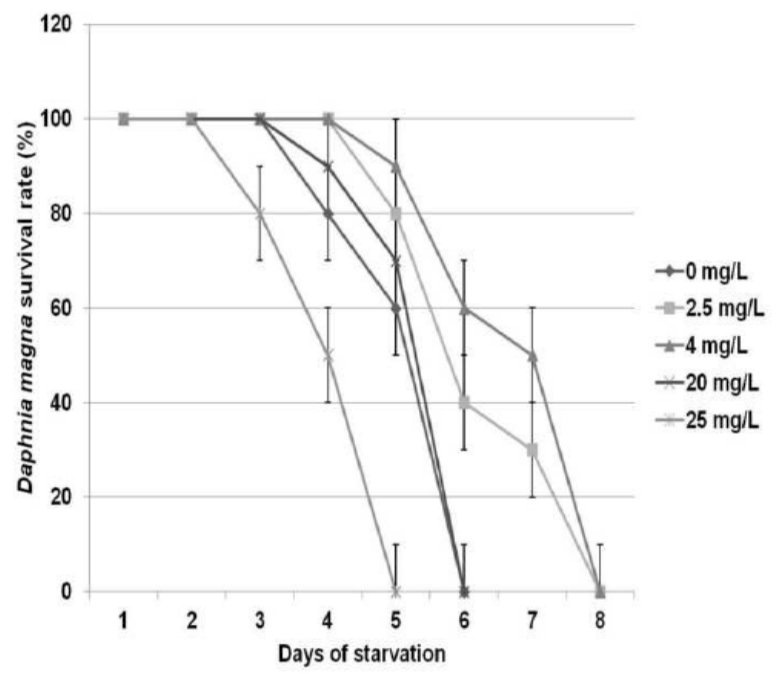

Figure 1: Survival rate of Daphnia magna neonates treated with different concentrations of ECT during starvation experiment. $\mathrm{n}=30$. 
Citation: Adam B, Zofia S (2015) Subchronic Effects of Ectoine on Survival, Growth and Physiological Parameters of Daphnia Magna. J Aquac Res Development 6: 352. doi:10.4172/2155-9546.1000352

by Tukey's test to detect differences among means. The analyses were completed using Develve statistical software. Values were statistically significant when $\mathrm{p}<0.05$.

\section{Results}

\section{Survival of daphnids during starvation experiment}

The non-treated daphnids subjected to starvation showed a decreased survival rate to $60 \pm 10 \%$ and $0 \%$ after 5 and 6 days, respectively (Figure 2). The lowest survival rate was observed at 25 $\mathrm{mg} / \mathrm{L}$ ECT $(50 \pm 10 \%$ and 4 and $0 \%$ after 5 days, respectively) when compared to the non-treated animals. On the other hand, less reduced survival rates showed animals exposed to the lower concentrations of ECT. The highest survival rate to be observed in daphnids exposed to 4 $\mathrm{mg} / \mathrm{L} \mathrm{ECT}$ with $90 \pm 9 \%$ at 5 day of the experiment.

\section{Swimming velocity}

The non-treated daphnids showed a rapid decrease of swimming velocity to $0.5 \pm 0.1 \mathrm{~mm} / \mathrm{s}$ after 5 days of starvation (Figure 3). Exposure to 20 and $25 \mathrm{mg} / \mathrm{L}$ ECT resulted in more rapid reduction of motility to $0.7 \pm 0.12 \mathrm{~mm} / \mathrm{s}$ after 5 days and $0.8 \pm 0.08 \mathrm{~mm} / \mathrm{s}$ after 3 days, respectively. On the other hand, daphnids treated with the lower concentrations of ECT showed less reduced swimming velocity however the values were lower when compared to the untreated group. Increased motility was noted in fed and ECT-exposed microcrustaceans (Figure 4). The most significant stimulation was manifested after $24 \mathrm{~h}$ in the group exposed to 20 and $25 \mathrm{mg} / \mathrm{L}$ ECT $(5.63 \pm 0.5 \mathrm{~mm} / \mathrm{s}$ and $5.67 \pm 0.54 \mathrm{~mm} / \mathrm{s}$, respectively) and it was maintained to the end of the experiment.

a)

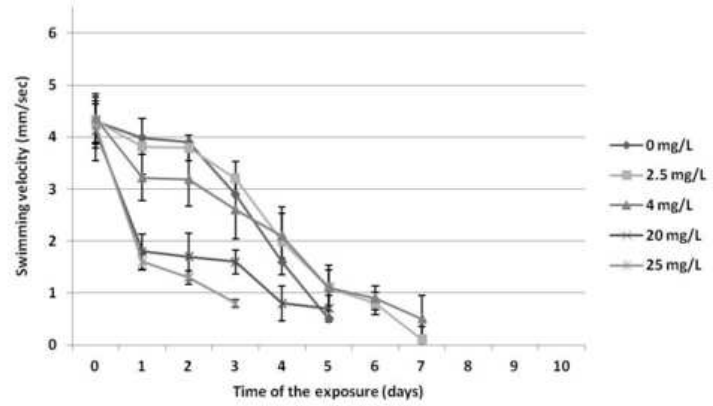

b)

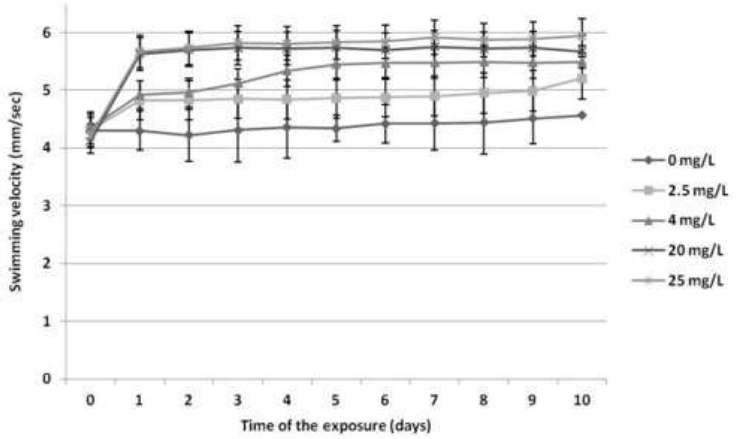

Figure 2: Swimming velocity of Daphnia magna exposed to different concentrations of ECT during starvation experiment (panel a) and normal feeding (panel b). $n=30$. a)

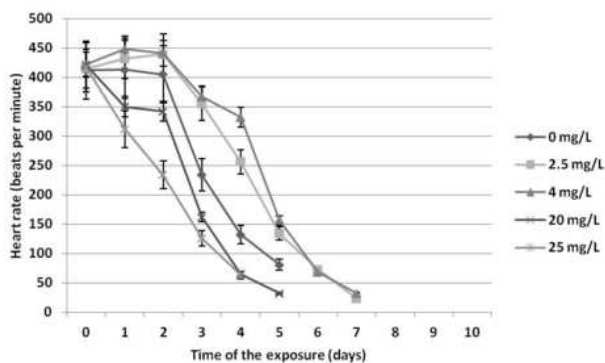

b)

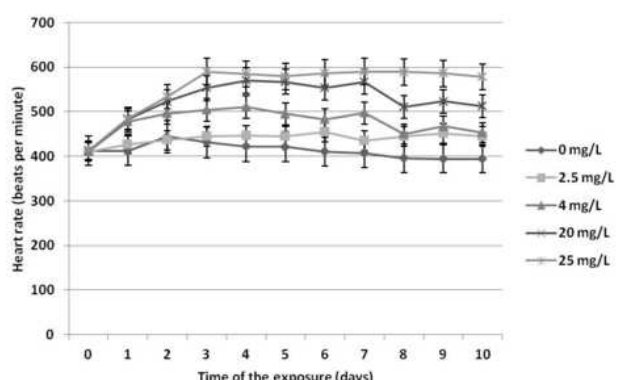

Figure 3: Heart rate of Daphnia magna treated with different concentrations of ECT during starvation experiment (panel a) and feeding with normal diet (panel b). $n=30$.

a)

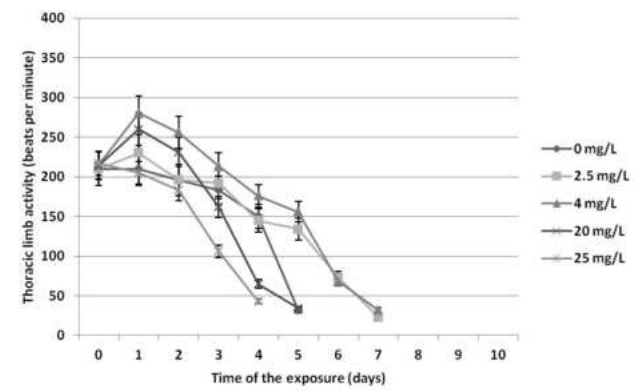

b)

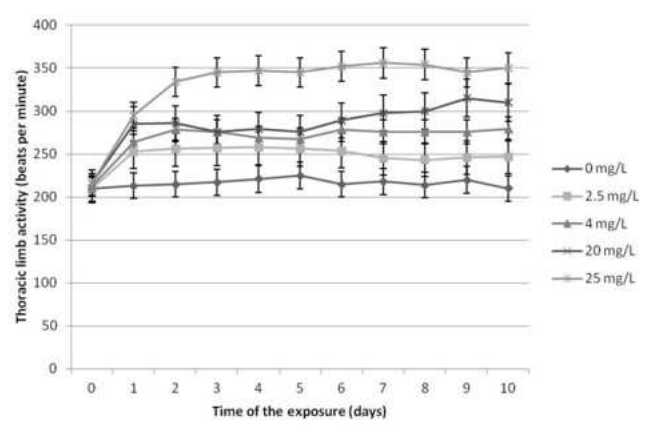

Figure 4: Thoracic limb activity of Daphnia magna exposed to various concentrations of ECT during starvation experiment (panel a) and feeding with normal diet (panel b). $n=30$. 


\section{Heart rate}

Starvation of the unexposed daphnids resulted in a continuous decrease of heart rate to $81 \pm 7 \mathrm{bpm}$ after 5 days (Figure 5). Daphnids treated with 20 and $25 \mathrm{mg} / \mathrm{L}$ ECT showed in more rapid inhibition (63 $\pm 5 \mathrm{bpm}$ after 5 days and $32 \pm 2 \mathrm{bpm}$ after 4 days, respectively). On the other hand, daphnids exposed to 2.5 and $4 \mathrm{mg} / \mathrm{L}$ ECT manifested initial increase of heart rate to $432 \pm 14 \mathrm{bpm}$ and $448 \pm 16 \mathrm{bpm}$ after 1 day and subsequent reduction, however it was attenuated when compared to the control. Fed daphnids that were treated with ECT showed a stimulated heart rate when compared to the untreated group (Figure 6). The highest value were noted in daphnids exposed to $25 \mathrm{mg} / \mathrm{L}$ ECT on 3 day of the experiment $(590 \pm 15 \mathrm{bpm})$ and it did not change significantly until the end of the experiment.

\section{Thoracic limb activity}

Starvation of daphnids resulted in a distinct decrease of thoracic limb activity of the unexposed group after 5 days to $32 \pm 9 \mathrm{bpm}$ and that treated with $20 \mathrm{mg} / \mathrm{L} \mathrm{ECT} \mathrm{(} 34 \pm 5 \mathrm{bpm}$ ) ( Figure 7). On the other hand, the animals treated with the lower concentrations of the osmoprotectant ( 2.5 and $4 \mathrm{mg} / \mathrm{L}$ ) manifested initial stimulation to $230 \pm 19 \mathrm{bpm}$ and $280 \pm 20 \mathrm{bpm}$ after $24 \mathrm{~h}$, respectively and subsequent inhibition which was more attenuated than those in the unexposed group. In contrast, the osmoprotectant induced stimulation of thoracic limb activity in fed daphnids (Figure 8). A substantial increase the activity was noted after 2 days of the exposure ( $334 \pm 22 \mathrm{bpm})$ and remained unchanged to the end of the experiment.

\section{Mandible movement rate}

Starvation resulted in a significant decrease of mandible movement rate of both ECT-treated and unexposed daphnids (Figure 9) with

a)

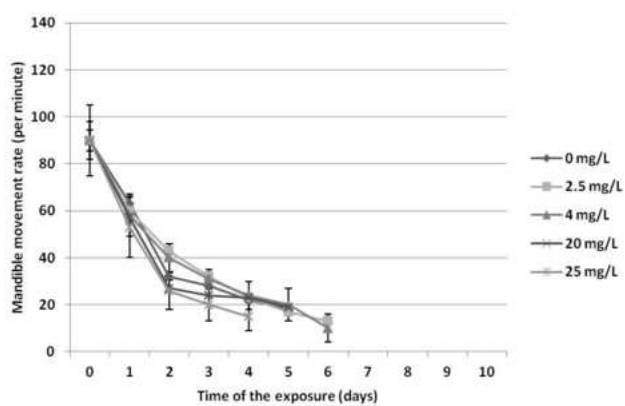

b)

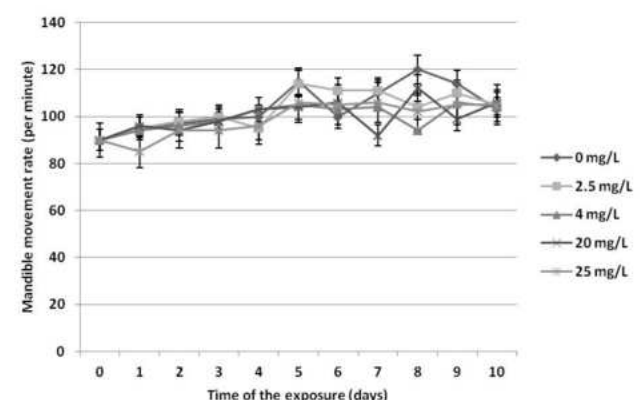

Figure 5: Mandible movement rate of Daphnia magna exposed to different concentrations of ECT during starvation experiment (panel a) and feeding with normal diet (panel b). $n=30$.

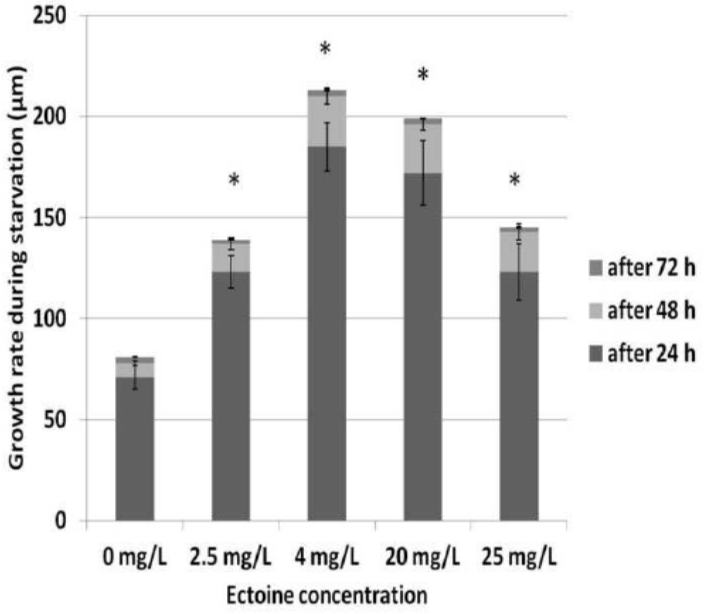

Figure 6: Effects of ectoine on growth rate of Daphnia magna during starvation experiment, $n=30$, *-statistical significance after $72 \mathrm{~h}$-exposure.

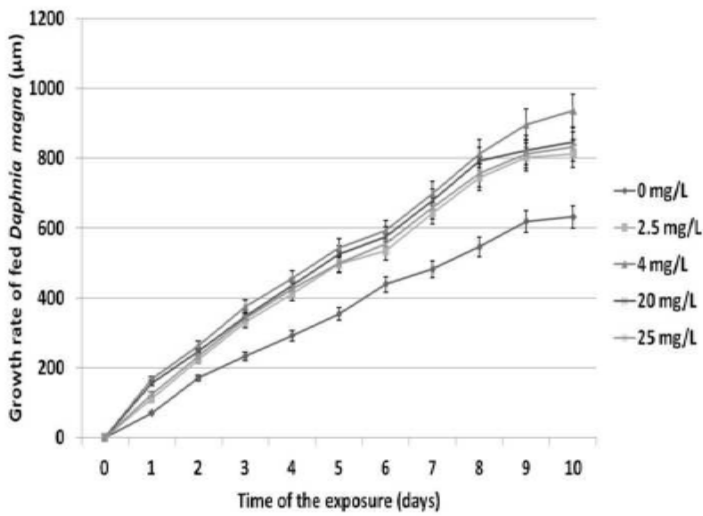

Figure 7: Growth rate of fed Daphnia magna exposed for 10 days to different concentrations of ectoine. $n=30$.

only a slight attenuation seen at 2.5 and $4 \mathrm{mg} / \mathrm{L}$ ECT. There were no significant changes of mandible movement rate in fed and ECTexposed daphnids when compared to the untreated crustaceans.

\section{Growth rate of Daphnia magna}

After the first 24-hours of the exposure all concentrations of ECT induced stimulation of growth of starving daphnids in comparison to the non-treated group (Figure 7). The most prominent increase of length was noted at 4 and $20 \mathrm{mg} / \mathrm{L}$ of the amino acid. After 48 hours daphnids the growth was significantly inhibited and its almost complete ceasation was observed after 72 hours of starvation experiment.

Fed and ECT-exposed daphnids showed increased growth rate when compared to the unexposed group (Figure 8). Although the highest stimulation was seen at $4 \mathrm{mg} / \mathrm{L}$ ECT there was no very significant diferences between the experimental groups of animals treated with ECT.

\section{Daphnia magna moulting}

$70 \pm 10 \%$ of the non-treated Dapnia magna showed moulting 
a)

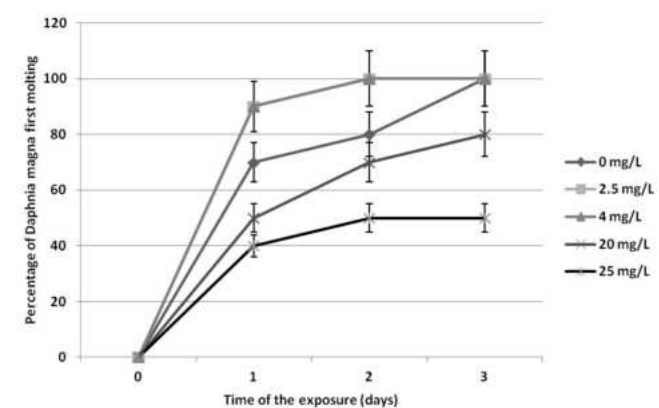

b)

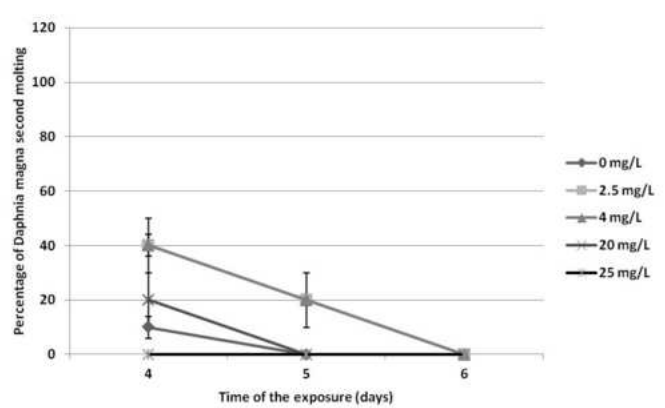

Figure 8: Effects of ectoine on moulting success of Daphnia magna during starvation experiment. Panels $a$ and $b$ show percentage of daphnids with first and second moulting, respectively. $n=30$.

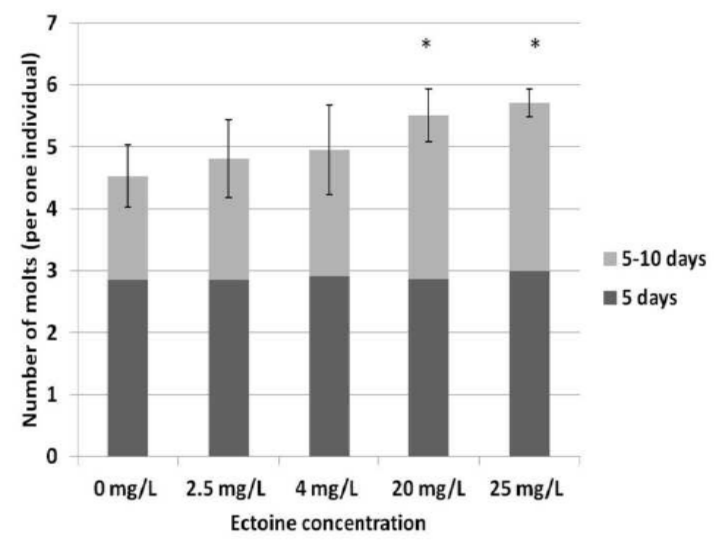

Figure 9: Number of moults of Daphnia magna fed with normal diet and exposed to different concentrations of ectoine, $n=30$ *-statistical significance.

success after $24 \mathrm{~h}$ (Figure 9). On the other hand, higher percentage (90 $\pm 10 \%$ ) showed daphnids exposed to the lowest concentrations 2.5 and $4 \mathrm{mg} / \mathrm{L}$ ECT Inhibition of ecdysis was found in the animals treated with 20 and $25 \mathrm{mg} / \mathrm{L}$ ( $40 \pm 6 \%$ and $50 \pm 10 \%$, respectively). The moulting success was decreased with increasing time of starvation to $10 \pm 5 \%$ in the untreated animals and $20 \pm 10 \%$ and $0 \%$ in daphnids exposed to 20 and $25 \mathrm{mg} / \mathrm{L}$.

No significant differences of moulting were found in fed daphnids after 5 days of exposure to different concentration of ECT. However after 10 days of the exposure, the number of moults increased in daphnids treated with 20 and $25 \mathrm{mg} / \mathrm{L} \mathrm{ECT}(5.51 \pm 0.43$ and $5.71 \pm 0.23$ moults per one individual, respectively) when compared to the control $(4,53 \pm 0.5)$.

\section{Discussion}

Organic osmolytes are osmoprotective molecules which may be accumulated at high concentrations without any interference with cellular processes [21]. Although ECT was found to be synthesized only by bacteria, its protective effects against various stressors were seen in different types of mammalian cells [22] and cladocerans [17]. To our best knowledge we present the first data on sub chronic toxicity of ectoine and its effects on animal growth.

\section{Survival of Daphnia magna}

The present study revealed that the same concentrations of ECT that were well tolerated during short- term experiments [18] turned out to decrease survival of unfed daphnids during the subchronic exposure. This is probably a result of longer time of exposure to the osmoprotectant in addition to the increased sensitivity of starving animals to its toxic effects. On the other hand, daphnids exposed to lower concentrations of ECT showed higher survival rates when compared to the unexposed animals. The mechanisms underlying this effect is not known, however it may be hypothesized that although ECT is not an essential dietary amino acid, its smaller amounts are less toxic and also may serve as source of energy for starving animals. As some bacteria were found to use ECT as an energetic source [23], it is possible that daphnids may also exploit this osmoprotectant in the same way. Interestingly, we did not find any lethal effects of the osmoprotectant in normally fed daphnids and exposed to the highest concentration of ECT but a distinct stimulation of animal growth and increased heart rate and thoracic limb movement which is discussed below.

\section{Swimming velocity}

Swimming velocity is a sensitive behavioral biomarker indicating health status of aquatic organisms because even slight toxicity may be reflected by alterations of the locomotor activity [24,25]. Glycine which is an amino acid and compatible solute was found to decrease locomotor activity of invertebrates [26]. In our previous short-term experiment we found the inhibitory effects of higher concentrations of ECT [18]but the motility of daphnids was hardly affected at its lower concentrations. The present study showed that the subchronic exposure of unfed daphnids to the same concentrations of ECT decreased swimming velocity in a concentration-dependent manner which may result, from animal starvation potentiating the effects induced by the highest concentrations used in the study. The lower concentrations of ECT attenuated the inhibition of unfed daphnid motility seen after 72 hours which may be explained by exploiting ECT as energy source. Our present study also revealed that fed crustaceans which were treated with ECT manifested a concentration-dependent increase of swimming speed and it was maintained elevated until the end of the experiment The stimulatory effect observed only in fed animals is probably a result of the increase of neuromuscular activity.

\section{Heart rate and thoracic limb movement}

Heart rate and thoracic limb activity of daphnids are common biomarkers used for determination of toxic effects induced by a variety of toxicants $[27,28]$. Our previous study showed modulatory effects of ECT on heart rate and thoracic limb activity of daphnids [18] and those findings were confirmed in the present study. Lower concentrations of ECT induced an initial increase of heart rate and thoracic limb 
movement in unfed daphnids, however the parameters were decreased at the higher concentrations. Other amino acids such as taurine and arginine were also found to modulate heart rate and inhibit arrhythmia in mammals [29]. Initial stimulation of thoracic limb movement of unfed daphnids may result from the metabolic stimulation induced by ECT and subsequent inhibition may be a consequence of energy depletion caused by starvation. On the other hand, fed daphnids showed a concentration-dependent, increase of both physiological parameters at each concentration of ECT and they remained unchanged to the end of the experiment.

\section{Mandible movement rate}

Mandible movement is one of physiological parameters used for determination of feeding activity of Daphnia magna [30-32]. Our results indicate that starvation resulted in a decreased mandible movement rate both in ECT-exposed and control daphnids probably caused by energy depletion in starving animals. Slight attenuation was noted only at the lower concentrations of the osmoprotectant. However, no differences between experimental groups were seen in fed animals indicating that this osmoprotectant does not affect this physiological parameter.

\section{Growth rate}

Changes in growth of daphnids are caused by different factors, such as food availability, predation and toxicants [33-36]. Taurine was found to be a stimulator of growth in marine moluscs [37]. The present study showed that ECT stimulates growth of Daphnia magna however the effect is dependent on the presence of food. The growth rate of unfed daphnids was much higher during first 24 hours than those in the group of unexposed animals. However, after 48 hours they were substantially decreased and it was almost completely ceased after 72 hours probably because of food depletion. Growth inhibition of unfed daphnids previously demonstrated by Bradley et al. may be a result of allocation of resources to essential for survial.

We chose a 10-day period for determination of the most significant alterations of growth rate between ECT-treated daphnids [38]. Here we demonstrate that fed daphnids subjected to ECT increased their growth rate when compared to the untreated group, however the stimulatory effect was not concentration-dependent. The most significant increase of growth was noted at $4 \mathrm{mg} / \mathrm{L}$ of ECT but higher amounts of the osmoprotectant were less effective. This may be a result of lower bioavailability of nutrients at higher concentrations of the osmoprotectant which may form a layer on cell membranes which is less permeable for nutrients. It is an open question whether stimulation of growth is induced by ECT itself which serves as an additional food source for Daphnia magna or by its enhancement of cell membrane permeability in different tissues resulting in the increase of food bioavailability.

\section{Moulting}

A lack of food and toxic effects induced by some chemicals may inhibit growth of neonates and thereby decrease moulting frequency [39-42]. The present study revealed that the exposure to the lower concentrations of ECT resulted in less decreased percentage of moulting daphnids than that in the control group. The inhibition of moulting of the non-treated animals may be a result of starvation and toxicity of the highest concentration of ECT. On the other hand, slightly higher number of moulting daphnids exposed to the lower concentrations of ECT correlated with their higher growth rate. Fed daphnids in all the experimental groups showed moulting success, however slightly higher number of shed carapaces showed daphnids exposed to the highest concentration of ECT which may be a sort of their defensive response also occuring under other stressful conditions $[43,44]$.

In summary, the present study showed that subchronic exposure to ECT induce modulatory, effects on survival, physiological parameters and growth of unfed and fed daphnids. ECT stimulated growth rate in fed animals which suggests that this osmoprotectant may be considered as a potent growth stimulator in cladocerans.

\section{Acknowledgement}

The authors would like to thank Professor Tadeusz Skowroński for a word of advice.

\section{References}

1. Pastor JM, Bernal V, Salvador M, Argandoña M, Vargas C, et al. (2013) Role of central metabolism in the osmoadaptation of the halophilic bacterium Chromohalobacter salexigens. J Biol Chem 288: 17769-17781.

2. Roessler M, Müller V (2001) Osmoadaptation in Bacteria and Archaea: common principles and differences. Environ Microbiol 3: 743-754

3. Harishchandra RK, Wulff S, Lentzen G, Neuhaus T, Galla HJ (2010) The effect of compatible solute ectoines on the structural organization of lipid monolayer and bilayer membranes. Biophys Chem 150: 37-46.

4. Pastor JM, Salvador M, Argandoña M, Bernal V, Reina-Bueno M, et al. (2010) Ectoines in cell stress protection: uses and biotechnological production. Biotechnol Adv 28: 782-801.

5. Galinski EA, Pfeiffer HP, Truper HG (1985) 1,4,5,6-Tetrahydro-2-methyl-4pyrimidinecarboxylic acid. A novel cyclic amino acid from halophilic phototrophic bacteria of the genus Ectothiorhodospira. Eur J Biochem. 149: 135-139.

6. Wei YH, Yuan FW, Chen WC, Chen SY (2011) Production and characterization of ectoine by Marinococcus sp. ECT1 isolated from a high-salinity environment J Biosci Bioeng 111: 336-342.

7. Pflughoeft KJ, Kierek K, Watnick PI (2003) Role of ectoine in Vibrio cholerae osmoadaptation. Appl Environ Microbiol 69: 5919-5927.

8. Kuhlmann AU, Bremer E (2002) Osmotically regulated synthesis of the compatible solute ectoine in Bacillus pasteurii and related Bacillus spp. Appl Environ Microbiol 68: 772-783.

9. Ongagna-Yhombi SY, Boyd EF (2013) Biosynthesis of the osmoprotectant ectoine, but not glycine betaine,

10. is critical for survival of osmotically stressed Vibrio parahaemolyticus Cells Appl Environ Microbiol 79: 5038- 5049.

11. Lippert K, Galinski EA (1992) Enzyme stabilization by ectoine-type compatible solutes: protection against heating, freezing and drying. Appl Microbiol Biotechnol 37: 61-65.

12. Göller K, Galinski EA (1999) Protection of a model enzyme (lactate dehydrogenase) against heat, urea and freeze-thaw treatment by compatible solute additives. J Mol Cat B 7: 37-45.

13. Knapp S, Ladenstein R, Galinski EA (1999) Extrinsic protein stabilization by the naturally occuring osmolytes beta-hydroxyectoine and betaine. Extremophiles 3: 191-198.

14. Zhang L, Wang Y, Zhang C, Wang Y, Zhu D, et al. (2006) Supplementation effect of ectoine on thermostability of phytase. J Biosci Bioeng 102: 560-563.

15. Grein TA, Freimark D, Weber C, Hudel K, Wallrapp C, et al. (2010) Alternatives to dimethylsulfoxide for serum-free cryopreservation of human mesenchyma stem cells. Int J Artif Organs. 33:370-80.

16. Sun H, Glasmacher B, Hofmann N (2012) Compatible solutes improve cryopreservation of human endothelial cells. Cryo Letters 33: 485-493.

17. Smiatek J, Harishhandra RK, Rubner O, Galla H-J, Heuer A (2012) Properties of compatible solutes in aqueous solution. Biophys Chem 160: 62-68.

18. Bownik A, Stepniewska Z, Skowroński T (2014) Protective effects of ectoine on heat-stressed Daphnia magna. J Comp Physiol B 184: 961-976.

19. Bownik A, Stępniewska Z, Skowroński T (2015) Effects of ectoine on behavioural, physiological and biochemical parameters of Daphnia magna. 
Citation: Adam B, Zofia S (2015) Subchronic Effects of Ectoine on Survival, Growth and Physiological Parameters of Daphnia Magna. J Aquac Res Development 6: 352. doi:10.4172/2155-9546.1000352

Comp Biochem Physiol Part C, Toxicol Pharmacol 168: 2-10.

20. Kelly AP, Weed LL (1965) Taurine as a constituent of a bacterial cell wall. J. Biol. Chem. 240:2519-2523.

21. Matsunari, H, Takeuchi T, Takahashi M, Mushiake K (2005) Effect of dietary taurine supplementation on growth performance of yellowtail juveniles Seriola quinqueradiata. Fisher Sci 71: 1131-1135.

22. Yancey $\mathrm{PH}$ (2005) Organic osmolytes as compatible, metabolic and counteracting cytoprotectants in high osmolarity and other stresses. J Exp Bio 208: 2819-2830.

23. Graf R, Anzali S, Buenger J, Pfluecker F, Driller H (2008) The multifunctional role of ectoine as a natural cell protectant. Clin Dermato1 26: 326-333.

24. Vargas C, Jebbar M, Carrasco R, Blanco C, Calderón MI, et al. (2006) Ectoines as compatible solutes and carbon and energy sources for the halophilic bacterium Chromohalobacter salexigens. J Appl Microbiol 100: 98-107.

25. Oliveira C, Almeida J, Guilhermino L, Soares AM, Gravato C (2012) Acute effects of deltamethrin on swimming velocity and biomarkers of the common prawn Palaemon serratus. Aquat Toxicol 15: 124-125.

26. Silva C, Oliveira C, Gravato C, Almeida JR (2013) Behaviour and biomarkers as tools to assess the acute toxicity of benzo(a)pyrene in the common prawn Palaemon serratus. Mar Environ Res 90: 39-46.

27. Finger W (1983) Effects of glycine on the crayfish neuromuscular junctions. Pflugers Arch 397: 121-127.

28. Villegas-Navarro A, Rosas-LE, Reyes JL (2003) The heart of Daphnia magna: effects of four cardioactive drugs. Comp Biochem Physiol C Toxicol pharmacol 136: $127-134$.

29. Campbell AK, Wann KT, Matthews SB (2004) Lactose causes heart arrhythmia in the water flea Daphnia pulex. Comp Biochem Physiol B Biochem Mol Biol 139: 225-234.

30. Eby G, Halcomb WW (2006) Elimination of cardiac arrhythmias using oral taurine with L-arginine with case histories: Hypothesis for nitric oxide stabilization of the sinus node. Med Hypotheses 67: 1200-1204.

31. Burns CW (1968) Direct observations of mechanisms regulating feeding behavior of Daphnia, in lakewater. Int. Rev. Gesamten Hydrobiol 53: 83-100.
32. Young S, Palm M, Grover JP, McKee D (1997) How Daphnia cope with algae selected for inedibility in long- running microcosms. J Plankt Res 19: 391-397.

33. Ghadouani A, Pinel-Alloul B, Plath K, Codd GA, Lampert W (2004) Effects of Microcystis aeruginosa and purified microcystin-LR on the feeding behavior of Daphnia pulicaria. Limnol. Oceanogr 49: 666-679.

34. Black RW, Hairston, N.G. (1988) Predator driven changes in community structure. Oecologia 77: 468-479.

35. Benard MF (2004) Predator-induced phenotypic plasticity in organisms with complex life histories. Annu. Rev. Ecol. Evol. Syst. 35: 651-673.

36. Heugens EH, Tokkie LT, Kraak MH, Hendriks AJ, Van Straalen NM, Admiraal W (2006) Population growth of Daphnia magna under multiple stress conditions: joint effects of temperature, food, and cadmium. Environ Toxicol Chem 25: 1399-1407.

37. Carter MJ, Silva-Flores P, Oyanedel JP, Ramos-Jiliberto R (2013) Morphological and life-history shifts of the exotic cladoceran Daphnia exilis in response to predation risk and food availability. Limnologica 43: 203-209.

38. Welborn J, Manahan D (1995) Taurine metabolism in larvae of marine invertebrate molluscs (Bilvalvia, Gastropoda). J Exp Biol 198: 1791-1799.

39. Stark JD, Vargas RI (2005) Toxicity and hazard assessment of fipronil to Daphnia pulex. Ecotoxicol Environ Saf 62: 11-16.

40. Zou E, Fingerman M (1997) Effects of estrogenic xenobiotics on molting of the water flea, Daphnia magna. Ecotoxicol Environ Saf. 38: 281-285.

41. Baldwin WS, Bailey R, Long KE, Klaine S (2001) Incomplete ecdysis is an indicator of ecdysteroid exposure in Daphnia magna. Environ Toxicol Chem. 20: 1564-1569

42. Dabrunz A, Duester L, Prasse C, Seitz F, Rosenfeldt R, et al. (2011) Biological surface coating and molting inhibition as mechanisms of $\mathrm{TiO} 2$ nanoparticle toxicity in Daphnia magna. PLoS ONE 6: e20112.

43. Rohrlack T, Christoffersen K, Kaebernick M, Neilan BA (2004) Cyanobacteria protease inhibitor microviridin $\mathrm{J}$ causes a lethal molting disruption in Daphnia pulicaria. Appl Environ Microbiol 70: 5047-5050.

44. Duneau D, Ebert D (2012) The role of moulting in parasite defence. Proc Biol Sci. 279: 3049-3054.

45. Shimizu N, Ogino C, Kawanishi T, Hayashi Y (2002) Fractal analysis of Daphnia motion for acute toxicity bioassay. Environ Toxicol 17: 441-448. 